\title{
Resistance Pattern and Genetics of Erythromycin-resistant Mutants of Streptococcus pyogenes
}

\author{
By H. MALKE \\ German Academy of Sciences, Institute of Microbiology and \\ Experimental Therapy, Jena 69, G.D.R
}

(Accepted for publication 29 October 1970)

SUMMARY

Single-step erythromycin-resistant strains of Streptococcus pyogenes 56I88 fell into two classes: whereas class A mutants were sensitive to lincomycin and carried mutations transducible by phage A 25, class B mutations conferred lincomycin cross-resistance and failed to be transducible under the conditions employed. Recombination and cotransduction experiments allowed the assigning of at least 10 of $\mathrm{I} 3$ class $\mathrm{A}$ mutations to the same linkage group. From interclass crosses recombinants emerged at frequencies comparable with those at which the donor markers were integrated into wild type. The resistance to three macrolides and lincomycin of interclass recombinants, carrying mutations which were not cotransducible, was related to the resistance levels determined by the individual mutations. The phenotypic properties of the ery mutants together with their genetic relationship lead to the conclusion that there are at least two loci governing resistance to erythromycin in strain $56 \mathrm{I} 88$ of $S$. pyogenes.

\section{INTRODUCTION}

The rapid emergence of penicillin-resistant strains of Staphylococcus aureus provided a stimulus for studying its susceptibility and the development of resistance to the macrolide antibiotics and lincomycin. These investigations brought to light a complex resistance pattern of individual mutant strains ranging from resistance to one and susceptibility to other macrolides (the so-called dissociated type of resistance) to complete cross-resistance to many macrolides and lincomycin (Jones, Nichols \& Finland, I956; Garrod, 1957; Pattee \& Baldwin, 1962; Barber \& Waterworth, I964; Duncan, I968; McGehee, Barrett \& Finland, I969; Weisblum \& Demohn, 1969).

Information on the corresponding situation in streptococci is sparse; nevertheless, this subject deserves consideration not only from a comparative point of view but also from a clinical standpoint (Paterson \& Madden, 1969; Griffith \& Black, 1970). This paper presents experiments aimed at isolating a number of erythromycin-resistant mutants of a group A streptococcal strain, elaborating their resistance pattern for erythromycin, carbomycin, oleandomycin and lincomycin, and establishing their genetic relationship. Efforts to elucidate the genetic constitution of resistant isolates seem to be highly pertinent in arriving at meaningful conclusions regarding the level of resistance and the range of cross-resistance conferred by individual mutations, a point little appreciated in previous work. The availability, at last, of transducing group 
Table I. Streptococcus pyogenes strains used in this study

\begin{tabular}{|c|c|c|}
\hline Strain & Genotype* & Source \\
\hline $\begin{array}{l}56188 \text { derivatives } \\
56188\end{array}$ & str-I ery ${ }^{+}$ & $\begin{array}{l}\text { Leonard et al. }(1968) \\
\text { who called it } 56 \times 188 \mathrm{~s}^{\mathrm{R}}\end{array}$ \\
\hline SM IOO & str-I ery-100 ) & \\
\hline SM 200 & str-I ery-200 & \\
\hline SM 300 & str-I ery-300 & \\
\hline SM 400 & str-I ery-400 & \\
\hline SM 500 & str-I ery-500 & \\
\hline SM 600 & str-I ery-60o & NG mutagenesis of \\
\hline SM 700 & str-I ery-70o & 56188 \\
\hline SM 800 & str-1 ery-800 & \\
\hline SM900 & str-I ery-900 & \\
\hline SM 1000 & str-I ery-I000 & \\
\hline SM 2000 & str-I ery-2000 & \\
\hline SM 3000 & str-I ery-3000) & \\
\hline SMIOI & str-I ery-10oery-I ) & \\
\hline SM IO2 & str-1ery-100ery-2 & \\
\hline SM IO3 & str-1 ery-100ery-3 & \\
\hline SM IO4 & str-I ery-100 ery-4 & NG mutagenesis of \\
\hline SMIO5 & str-I ery-100 ery-5 & SMIOO \\
\hline SM 106 & str-I ery-100 ery-6 & \\
\hline SM 107 & str-1 ery-10o ery-7 & \\
\hline SM IO8 & str-1 ery-100 ery-8) & \\
\hline SM 109 & str-1 ery-100 ery-500 & SM $500 \times$ SM $100 \dagger$ \\
\hline SMIIO & str-I ery-200 ery-2000 & SM $200 \times$ SM 2000 \\
\hline SM4OI & str-I ery-10o ery-400 & SM $400 \times$ SM 100 \\
\hline SM402 & str-I ery-10oery- 600 & SM $600 \times$ SM 100 \\
\hline SM 403 & str-I ery-100 ery-700 & SM $700 \times$ SM 100 \\
\hline SM 404 & str-I ery-100 ery-800 & SM $800 \times$ SM 100 \\
\hline SM 405 & str-I ery-100 ery-900 & SM900 $\times$ SM 100 \\
\hline SM 406 & str- I ery-IOo ery-IO00 & SM $1000 \times$ SM 100 \\
\hline SM 407 & str-I ery-100 ery-3000 & SM $3000 \times$ SM 100 \\
\hline SM 408 & str-I ery-200 ery-400 & SM $400 \times$ SM 200 \\
\hline SM 409 & str-I ery-200 ery-600 & $\operatorname{SM} 600 \times \operatorname{SM} 200$ \\
\hline SM4IO & str-I ery-200 ery-700 & $\operatorname{SM} 700 \times \operatorname{SM} 200$ \\
\hline SM4I I & str-I ery-200 ery-800 & SM $800 \times \operatorname{SM} 200$ \\
\hline SM4II & str-I ery-200 ery-900 & SM $900 \times$ SM 200 \\
\hline SM4I3 & str-I ery-200 ery-1000 & SM $1000 \times \operatorname{SM} 200$ \\
\hline SM4I4 & str-I ery-200 ery-3000 & $\operatorname{SM} 3000 \times \operatorname{SM} 200$ \\
\hline SM415 & str-I ery-300 ery-400 & $\operatorname{SM} 400 \times \operatorname{SM} 300$ \\
\hline SM 416 & str-1 ery-300 ery-600 & $\operatorname{SM} 600 \times \operatorname{SM} 300$ \\
\hline SM4I7 & str-I ery-300 ery-700 & SM $700 \times$ SM 300 \\
\hline SM 418 & str-I ery-300 ery-800 & SM $800 \times \operatorname{SM} 300$ \\
\hline SM 419 & str-I ery-30o ery-900 & SM $900 \times$ SM 300 \\
\hline SM 420 & str-I ery-300 ery-1000 & SM $1000 \times$ SM 300 \\
\hline SM42I & str-1 ery-300 ery-3000 & $\operatorname{SM} 3000 \times \operatorname{SM} 300$ \\
\hline SM 422 & str-I ery-500 ery-400 & SM $400 \times$ SM 500 \\
\hline $\operatorname{SM} 423$ & str-1 ery-500 ery-60o & SM $600 \times \operatorname{SM} 500$ \\
\hline SM 424 & str-I ery-500 ery-700 & SM $700 \times$ SM 500 \\
\hline SM 425 & str-I ery-500 ery-800 & SM $800 \times$ SM 500 \\
\hline SM 426 & str-I ery-500 ery-900 & SM900 $\times$ SM 500 \\
\hline SM 427 & str-I ery-50o ery-1000 & SM $1000 \times$ SM 500 \\
\hline SM 428 & str-I ery-500 ery-3000 & SM $3000 \times$ SM 500 \\
\hline SM 429 & str-I ery-2000 ery-400 & SM $400 \times$ SM 2000 \\
\hline SM430 & str- $x$ ery-2000 ery-600 & $\operatorname{SM} 600 \times$ SM 2000 \\
\hline SM43I & str-I ery-2000 ery-700 & SM $700 \times$ SM 2000 \\
\hline SM 432 & str-I ery-2000 ery-800 & SM $800 \times$ SM 2000 \\
\hline
\end{tabular}


Table I (cont.)

\begin{tabular}{lll} 
Strain & \multicolumn{1}{c}{ Genotype* } & \multicolumn{1}{c}{ Source } \\
SM 433 & str-I ery-2000 ery-900 & SM $900 \times$ SM 2000 \\
SM 434 & str-I ery-2000 ery-1000 & SM I000 $\times$ SM 2000 \\
SM 435 & str-I ery-2000ery-3000 & SM 3000 $\times$ SM 2000 \\
K 56 derivatives & & \\
K 56 & str ${ }^{+}$ery ${ }^{+} u v r^{+}$ & Kjems (1958) \\
SM 40 & $u v r-40$ & NG mutagenesis of \\
SM 60 & $u v r-60$ & K56 (Malke, 1967) \\
SM 63 & $u v r-63$ &
\end{tabular}

* In genetic nomenclature the proposals of Demerec, Adelberg, Clark \& Hartman (1966) were followed. Abbreviations: str, ery and $u v r$ symbolize loci determining response to streptomycin, erythromycin and ultraviolet radiation, respectively. The mutation conferring high-level resistance to streptomycin on strain 56I 88 was designated str-r. Site descriptions ery-400, ery-600 to ery-rooo, and ery-3000 are tentative.

$\dagger$ Transductions are indicated as recipient $\times$ donor.

A streptococcal bacteriophages (Leonard, Colón \& Cole, I968; Malke, I969, 1970) enables an approach to this problem to be made and the data presented show that phage A 25, used throughout this work, is a useful tool in genetic studies.

\section{METHODS}

Organisms. Two basic streptococcal strains were used, 56188 (group A, type 6) and $\mathrm{K} 56$ (group A, type 12). Genetic markers were obtained in these strains following mutagenesis with $N$-methyl- $N^{\prime}$-nitro- $N$-nitrosoguanidine (NG). Table I gives the genotypes and derivations of the strains used.

Media. The composition of serum broth (SB), serum agar (SA) and soft serum agar (SSA) has been described by Malke (1969).

Chemicals. Erythromycin, carbomycin and oleandomycin (Pfizer, Brooklyn, New York); lincomycin (Upjohn, Kalamazoo, Michigan, U.S.A.); streptomycin (Jenapharm, Jena); $N$-methyl- $N^{\prime}$-nitro- $N$-nitrosoguanidine (Ferak, Berlin, Germany).

Isolation of erythromycin-resistant mutants. Exponentially growing cultures of $56 \mathrm{I} 88$ ( $10^{8}$ to $5 \times 10^{8}$ colony-forming units $/ \mathrm{ml}$. in SB) were centrifuged and resuspended in $0.15 \mathrm{M}$-acetate buffer at $\mathrm{pH} 5.0$ containing $100 \mu \mathrm{g}$. $/ \mathrm{ml}$. of NG. After incubation for 20 to $30 \mathrm{~min}$. at $37^{\circ}$ the cells were washed once with phosphate buffer, $\mathrm{pH} 7^{\circ}$, and resuspended in SB at the original density. After regrowing the resulting suspensions for 5 to $\mathrm{I} 6 \mathrm{~h}$. at $37^{\circ}, 0 . \mathrm{I} \mathrm{ml}$. samples were plated on SA containing $0.1 \mu \mathrm{g} . / \mathrm{ml}$. of erythromycin. Colonies which appeared after 2 days of incubation were isolated, purified by repeated single colony isolation and retested to confirm their response. Of the I 2 single-step erythromycin-resistant strains isolated in this way, mutant SM 100 was in turn used to obtain, after selection at $2 \mu \mathrm{g} . / \mathrm{ml}$. of erythromycin, the eight second-step resistant mutants SM IOI to SM I08. All mutant strains listed in Table I were of independent mutational origin. They were lyophilized and during the experiments care was taken to prevent possible enrichment of sensitive revertants by repeated reisolation of single colonies from SA containing appropriate amounts of erythromycin.

Determination of resistance to antibiotics. The maximum level of resistance to the 
three macrolides and lincomycin of the isolated strains was estimated by streaking loopfuls of bacterial suspensions grown overnight in SB on to SA plates containing serial concentrations of the respective antibiotics. After incubation at $37^{\circ}$ for $24 \mathrm{~h}$. the minimal inhibitory concentrations defined as the lowest antibiotic concentrations preventing confluent growth of the strains were recorded. Certain strains were reexamined up to four times on different dates and the variations in minimal inhibitory concentrations, if any, did not exceed $50 \%$.

Transduction. The phage strain used in transduction was the double temperaturesensitive mutant, ts I-2, of the virulent phage A 25; this prevents killing of transduced cells by non-defective phage when transduction is performed at the restrictive temperature (Malke, 1969). Transducing lysates were prepared by the agar-layer technique (Adams, I959) at $29.5^{\circ}$. In transductions by the agar-layer plating technique, $0 \cdot$ I to $0.3 \mathrm{ml}$. portions of stationary phase recipient cells in SB were mixed with equal volumes of phage at multiplicities of infection ranging from 0.1 to 0.7 phage/cell. After $20 \mathrm{~min}$. adsorption at $37.5^{\circ}$ the mixtures received $3 \mathrm{ml}$. of SSA and were poured over SA plates (I I ml./plate). These were incubated for $2 \mathrm{~h}$ at $37.5^{\circ}$ and then overlaid with $8 \mathrm{ml}$. of SSA containing, respectively, $750 \mu \mathrm{g} . / \mathrm{ml}$. of streptomycin and appropriate challenging concentrations of erythromycin. Transduced colonies were counted after further incubation for $48 \mathrm{~h}$. at $37.5^{\circ}$. In the direct plating technique, $\mathrm{I} \cdot 2 \mathrm{ml}$. of fresh SB containing antiphage serum at a final $\mathrm{K}$ value of about $\mathrm{r}$ was added to $0.6 \mathrm{ml}$. mixtures of recipient cells and phage after adsorption. After $3 \mathrm{~h}$. of incubation at $37.5^{\circ}, 0 . \mathrm{I} \mathrm{ml}$. samples were spread on to SA plates containing, respectively, $200 \mu \mathrm{g} . / \mathrm{ml}$. of streptomycin and appropriate concentrations of erythromycin.

Determination of recombination between linked markers. To avoid variables such as differences in specific transducing activities of various lysates, an unrelated marker (str-I) present in all donor strains served as a standard of reference. The ratio of ery- $x$ to str-I cells in a cross $e r y^{+} s t r^{+} \times e r y-x$ str- $I$ was taken as unity and the recombination index (RI) of a cross between two non-allelic erythromycin-resistant mutants, ery- $x$ and ery-y, was expressed as a fraction of this unit, according to the formula (Gunsalus, Gunsalus, Chakrabarty, Sikes \& Crawford, I968; Sirotnak \& Hachtel, 1969)

$$
\mathrm{RI}=\frac{e r y-y \times e r y-x \text { str }-I}{e r y^{+} s_{1}{ }^{+} \times e r y-x \text { str-I }}=\frac{e r y-x e r y-y / s t r-I}{e r y-x / \text { str }-I} .
$$

Determination of donor marker cotransduction. In a wild-type $\times$ double-mutant cross the frequency of cotransfer (cotransfer index, CI) of the two donor markers was expressed as the proportion of donor-type transductions among the total transductions, according to

$$
\mathrm{CI}=\operatorname{ery} y^{+} \times \operatorname{ery}-x \operatorname{er} y-y=\operatorname{ery}-x \operatorname{er} y-y /(\operatorname{ery}-x \operatorname{er} y-y+e r y-x+e r y-y) .
$$

\section{RESULTS}

\section{Resistance to macrolides and lincomycin of the mutants isolated}

The results in Fig. I show the minimal inhibitory concentrations of each antibiotic for the strains studied. The difference between the wild-type level of erythromycin resistance and that of the single-step mutants ranged from four- to 20-fold and the second-step mutants were 80 - to 200 -fold more resistant than wild type or eight- to 

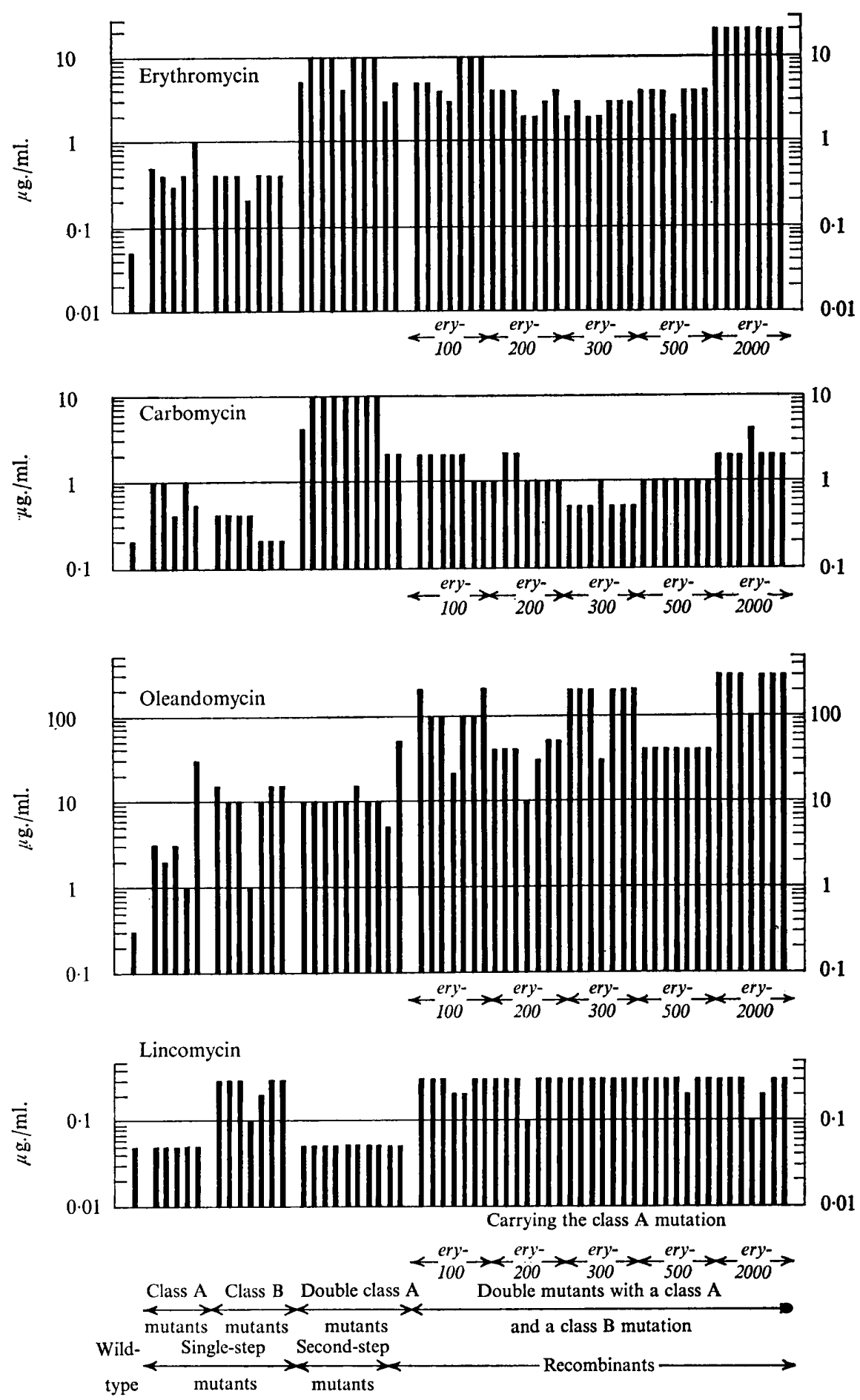

Fig. I. Resistance pattern of wild-type, erythromycin-resistant mutants and derived recombinants for three macrolides and lincomycin. The vertical bars indicate minimal inhibitory concentrations of the antibiotics. From left to right, the responses of the following strains are shown. Wild type, 56188; class A mutants, SM 100, SM 200, SM 300, SM 500, SM 2000; class B mutants, SM 400, SM 600, SM 700, SM 800, SM 900, SM I000, SM 3000; doubleclass A mutants, SM IOI to SM IIO; double mutants with a class A and a class B mutation, SM 4OI to SM 435. The genotypes of these strains are given in Table 1. 
20-fold more resistant than SM IOO from which they were derived. Note that the most sensitive second-step mutant had a maximum level of erythromycin resistance four times higher than that of the most resistant single-step mutant. While all of the single-step mutants were found to be cross-resistant to oleandomycin at different degrees, they may or may not be resistant to carbomycin and lincomycin. This shows that strains doubly resistant to erythromycin and oleandomycin may well be sensitive to carbomycin, and this type of resistance might be termed 'partially dissociated'. The second-step mutants showed a rather uniform behaviour towards carbomycin and oleandomycin, all being inhibited at higher concentrations than their parent strain, sMroo. The most remarkable feature of the second-step mutants was their unaltered response to lincomycin: they were as susceptible to this antibiotic as were both parent strain and wild type.

\title{
Transduction efficiencies of ery mutations carried by the single-step mutants
}

Table 2 compares the frequency of transduction of each ery mutation to the frequency of transduction of the standard reference marker, str- $r$, using SM 60 and 56 I 88 as recipients in heterologous and homologous crosses. The individual transduction

\section{Table 2. Transduction efficiencies of various ery mutations}

\begin{abstract}
Transduction efficiencies are calculated from the ratio of ery to $s t r-r$ transductants and represent means \pm standard deviations derived from 3 to 4 replicate determinations. Since 56188 is streptomycin-resistant, transduction efficiencies are given in terms of SM60-derived str-I transductions. Experimental conditions: agar layer plating technique; multiplicity about $0 . \mathrm{I}$; erythromycin concentration of top agar, $0.5 \mu \mathrm{g} . / \mathrm{ml}$.
\end{abstract}

\begin{tabular}{lcc} 
& \multicolumn{2}{c}{ Recipient } \\
DM 60 & 56188 \\
SM 100 & $0.55 \pm 0.04$ & $0.56 \pm 0.08$ \\
SM 200 & $0.67 \pm 0.12$ & $0.74 \pm 0.16$ \\
SM 300 & $0.50 \pm 0.03$ & $0.44 \pm 0.01$ \\
SM 500 & $0.84 \pm 0.15$ & $0.92 \pm 0.10$ \\
SM 2000 & $1.02 \pm 0.24$ & $0.93 \pm 0.09$ \\
SM 400 & $<0.002$ & $<0.002$ \\
SM 600 & $<0.005$ & $<0.005$ \\
SM 700 & $<0.005$ & $<0.005$ \\
SM 800 & $<0.003$ & $<0.003$ \\
SM900 & $<0.002$ & $<0.002$ \\
SM 1000 & $<0.002$ & $<0.002$ \\
SM 3000 & $<0.005$ & $<0.005$ \\
56188 & $<0.003$ & $<0.003$
\end{tabular}

efficiencies fell into two classes: class A, comprising mutations which were transduced at 50 to $100 \%$ the efficiency of str-I, and class B, comprising mutations which were transduced at efficiencies which did not exceed the control values using 56r88 as donor. Fig. I reveals the remarkable fact that the non-transducibility of class $B$ mutations coincides with their property of conferring cross-resistance to lincomycin.

When the experiment given in Table 2 was carried out by the direct plating technique at a challenging erythromycin concentration of about $0.075 \mu \mathrm{g} . / \mathrm{ml}$. the essential features of Table 2 could be reproduced, showing that neither prolonged incubating for phenotypic expression nor the low concentration of erythromycin (less than 
resistance level of donors) resulted in transducibility of class B mutations. Furthermore, the failure to demonstrate transduction was not due to the general inability of class B mutants to function as genetic donors, since transducing particles bearing str- $\boldsymbol{I}$ were obtained at normal frequencies from each class B strain. Replica-plating experiments to test class A transductants for susceptibility to carbomycin and oleandomycin revealed that all of the transduced colonies carrying a particular class A mutation were uniformly resistant to the appropriate concentrations of these antibiotics, indicating that each class A mutation was transferred as a discrete unit, all transductants being of the donor type. Replica-plating also showed that none of the class A mutations was cotransduced with the $s t r-I$ marker.

\section{Genetic constitution of the second-type mutants}

The stepwise construction of the second-step mutants suggested that they represent double mutants carrying a second mutation in addition to the ery-Ioo marker, the two mutations acting synergistically. Evidence in support of this assumption came

\section{Table 3. Joint transduction of mutations carried by the second-step mutants}

The linkage values represent means \pm standard deviations derived from transductions using four different recipient strains, K 56, SM 40, SM 60 and SM 63. Experimental conditions: agarlayer plating technique; multiplicity about 0.7 ; top agar contained $6 \mu \mathrm{g} . / \mathrm{ml}$. of erythromycin for the selection of double marker transductants and $0.6 \mu \mathrm{g} . / \mathrm{ml}$. for the selection of total transductants.

$\begin{array}{lclc}\text { Donor } & \text { Cotransfer index } & \text { Donor } & \text { Cotransfer index } \\ \text { SM IOI } & 0.87 \pm 0.03 & \text { SM IO6 } & 0.78 \pm 0.05 \\ \text { SM IO2 } & 0.83 \pm 0.01 & \text { SM IO7 } & 0.75 \pm 0.06 \\ \text { SM I O3 } & 0.82 \pm 0.05 & \text { SM IO8 } & 0.83 \pm 0.03 \\ \text { SM I04 } & 0.76 \pm 0.02 & \text { SM IOO } & <0.003 \\ \text { SM I05 } & 0.89 \pm 0.06 & \text { (control) } & \end{array}$

from experiments showing that the mutations presumed to be borne by these strains were separable by recombination (Table 3). Phage propagated on a second-step mutant strain, in addition to producing donor-type transductants, gave rise to a class of transduced cells with a lower level of resistance; these turned out to carry the separated markers individually. The fact that double marker transductants could be obtained at all means that the mutational sites were linked closely enough to be transduced jointly, the frequencies of joint transfer ranging from 75 to $89 \%$ (Table 3 ).

\section{Recombination between mutants carrying class A mutations}

Two-point crosses between the five class A mutants were performed, selecting possible recombinants with higher resistance levels. Table 4 contains the results of the ten crosses, together with the reciprocal crosses and selfing experiments, the latter serving as controls. In two crosses recombinants having the genotypes ery-Iooery-500 and ery-200ery-2000 were detected. Although two of the recipients showed a relatively high background of spontaneous mutants, the corresponding reciprocal crosses with the stable recipients gave unequivocal results, so that the outcome of these crosses can be considered established. Tests for cotransfer showed that ery-50o was cotransduced at a frequency of $46 \%$ with ery-IOo and ery-2000 at a frequency of $31 \%$ with ery-20o. The frequencies of cotransfer and the frequencies of recombination were inversely correlated. 
Fig. I shows that the double class A mutants constructed by recombination were similar to the second-step mutants in all essential aspects, including the possession of higher than single levels of resistance to erythromycin, carbomycin and oleandomycin and full sensitivity to lincomycin.

Table 4. Recombination indices in reciprocal two-point crosses between class $A$ mutants, together with the results of selfing experiments which served as controls

Recombination indices, as defined in Methods, represent averages \pm standard deviations based on 7 to 19 separate determinations. Experimental conditions: direct plating technique; recipient markers were present in a SM 60 background; in wild-type (SM 60) $\times$ mutant reference crosses, ery transductants were selected for at $0.1 \mu \mathrm{g} . / \mathrm{ml}$. of erythromycin; in mutant $\times$ mutant crosses the SA plates contained $1 \mu \mathrm{g} . / \mathrm{ml}$. of the antibiotic, with the exception of crosses involving ery-2000 in which $2 \mu \mathrm{g}$. $/ \mathrm{ml}$. were used.

$\begin{array}{llllll}\text { Recipient } & \overbrace{\text { ery-100 }} & \text { ery-200 } & \text { ery-300 } & \text { ery-500 } & \text { ery-2000 } \\ \text { ery-100 } & 0.050 \pm 0.017 & 0.065 \pm 0.027 & 0.046 \pm 0.023 & 0.144 \pm 0.014 & 0.044 \pm 0.019 \\ \text { ery-200 } & 0.007 & <0.007 & <0.008 & <0.009 & 0.550 \pm 0.068 \\ \text { ery-300 } & <0.007 & <0.007 & <0.007 & <0.007 & <0.005 \\ \text { ery-500 } & 0.231 \pm 0.059 & <0.012 & <0.008 & <0.010 & <0.007 \\ \text { ery-2000 } & 0.207 \pm 0.121 & 0.547 \pm 0.109 & 0.255 \pm 0.110 & 0.264 \pm 0.094 & 0.289 \pm 0.144\end{array}$

Table 5. Number of recombinants per plate from interclass crosses between class $A$ and class $B$ mutants

The numbers of colonies given are averages from two to three separate determinations. Experimental conditions: agar-layer plating technique; transduction mixtures consisted of about $3 \times 10^{8}$ recipient cells and $3 \times 10^{7}$ phages. Erythromycin concentration in the top agar was $6 \mu \mathrm{g} . / \mathrm{ml}$., except for crosses SM $800 \times$ SM 200 , SM $800 \times$ SM 300, SM $800 \times$ SM 500 and SM $1000 \times$ SM 500, in which $3 \mu \mathrm{g}$. $/ \mathrm{ml}$. were used. When 56188 was the recipient, transductants were selected for at $0.5 \mu \mathrm{g} . / \mathrm{ml}$. of erythromycin in the top agar.

$\begin{array}{lcccccc}\begin{array}{c}\text { Class B } \\ \text { recipient and } \\ \text { wild type }\end{array} & \overbrace{\text { SM I00 }} & \text { SM 200 } & \text { SM 300 } & \text { SM 500 } & \text { SM 2000 } & \text { Selfings } \\ \text { SM400 } & 376 & 602 & 557 & 421 & 302 & 8 \\ \text { SM 600 } & 403 & 305 & 384 & 500 & 361 & \text { II } \\ \text { SM 700 } & 265 & 366 & 432 & 262 & 202 & 3 \\ \text { SM 800 } & 134 & 27 \text { I } & 280 & 220 & \text { I29 } & 0 \\ \text { SM900 } & 303 & 273 & 247 & \text { I I0 } & 279 & \text { O } \\ \text { SM 1000 } & 279 & 267 & 225 & 327 & 169 & 3 \\ \text { SM 3000 } & 381 & 448 & 493 & 634 & 412 & 9 \\ \text { 56188 } & 373 & 433 & 304 & 446 & 294 & 0\end{array}$

\section{Recombination between class $A$ and class $B$ mutants}

While the non-transducibility of class $B$ mutations excluded corresponding intraclass B crosses, it was possible to perform all 35 interclass crosses, using the seven class B mutants as recipients. Recombinants, which again were selected on the basis of higher resistance to erythromycin, were readily detected in each cross at frequencies similar to those at which the donor markers were transferred to wild-type 56188 (Table 5). One transductant colony from each cross was chosen to establish the strains SM 40I to SM435 (Table I), which were considered to represent double mutants bearing a class A and a class B mutation. Each of these interclass recombinants was then used 
as a donor in transduction, using the direct plating technique, with SM 60 as recipient. Two different erythromycin concentrations were used which selected either for donor type transductants or total transductants. In no case could double marker transductants be detected; if they arose at all they did so at frequencies less than 0.7 to $0.2 \%$. This indicated the absence of cotransduction of class A and class B mutations. The colonies which appeared on the plates selective for total transductants were replicated on to plates containing lincomycin at concentrations which prevented class A transductants colonies from growing but would have allowed growth of transductants bearing class B mutations. In no case were developing colonies found on the replica plates, indicating once more the failure of class B mutations to be transduced.

Erythromycin-resistant class A and class B mutations when present together in the same cell acted synergistically rather than additively (Fig. I). Since the level of resistance to erythromycin of the class B mutants was rather uniform, the degree of resistance of interclass recombinants was largely determined by the individual class A mutation present. On the whole, there was no fundamental difference in erythromycin resistance between double class $\mathbf{A}$ and double mutants bearing a class $\mathbf{A}$ and a class $\mathbf{B}$ mutation, the average minimal inhibitory concentration being $7 \cdot 7$ and $7 \cdot 2 \mu \mathrm{g} / \mathrm{ml}$, respectively. The combined action of a class A and a class B mutation was also synergistic with respect to their contribution to oleandomycin cross-resistance. Since in this case class B mutants were already able to attain resistance levels similar to those shown by double class A mutants, interclass recombinants were considerably more resistant than intraclass $\mathrm{A}$ recombinants. On the other hand, the carbomycin resistance of interclass recombinants was lower than that of double class A mutants Finally, class A mutations did not contribute to lincomycin resistance and the resistance of interclass strains was accordingly that which was determined by class B mutations.

\section{DISCUSSION}

The single-step erythromycin-resistant mutants studied appear to fall into two classes which may clearly be distinguished both phenotypically and genetically. The failure of class B mutations to be transduced does not seem to be due to genetic incompatibility among donors and recipients since it applies to both homologous and heterologous transductions. Among several possibilities which might explain this behaviour are the exclusion of class B mutations from incorporation into transducing particles and a longer than $3 \mathrm{~h}$. period for phenotypic expression. Since at present these mutations cannot be handled genetically, the site descriptions of class B mutants given in Table I must remain tentative.

From recombination and cotransduction experiments it is evident that the class $\mathrm{A}$ mutations ery-I to ery-8, ery-I0o and ery-50o belong to the same linkage group, as defined by cotransfer with phage A 25. The remaining class A mutations ery-200, ery-300 and ery-200o probably belong to this linkage group also, but definitive proof is lacking. Recombination experiments involving ery-I to ery-8 might help to clarify this question. For this reason the non-committal term class A mutations is used rather than the locus designation, eryA.

The synergistic action of certain linked ery mutations has also been demonstrated in pneumoccocus by Ravin \& Iyer (196I). Such stepwise development of resistance is not restricted to the macrolide antibiotics but may also occur with a number of other 
antibacterial agents (Banic, 1959; Bryan, 1961; Rotheim \& Ravin, 196r ; Sirotnak \& Hachtel, 1969) and so may be the rule rather than the exception. Failure to detect recombinants in 8 of 10 intraclass $A$ crosses may be due either to the absence of recombination or to the inability of recombinants to express enhanced levels of resistance. Although no attempts were made to decide between these alternatives, this situation is in sharp contrast to the interaction of class A and class B mutations, which in each of the 35 possible combinations studied were capable of joint expression in recombinant genomes. What, then, is the genetic relationship of the mutations in such interclass recombinant strains? In view of the general inability of class B mutations to be transduced, it is unfortunately not possible to attach great importance to the absence of cotransduction of mutations belonging to different classes. Nevertheless, it seems unlikely that the locus harbouring class $\mathrm{A}$ mutations contains regions which are either specifically excluded from incorporation into transducing particles or fail to be integrated into the recipient genome. The fact that interclass recombinants occur at frequencies comparable with those at which the donor markers are integrated into wild type makes it reasonable to conclude that class A and class B mutations are not located within the same locus and that there are at least two loci governing resistance to erythromycin in strain 56188 of Streptococcus pyogenes.

Note added in proof. The five mutations ery-100, ery-200, ery-300, ery-500 and ery-2000 were found to be linked to two non-allelic mutations conferring resistance to lincomycin but not erythromycin. Thus all class A mutations studied belong to the same linkage group.

I wish to acknowledge the technical assistance of Mrs Irene Hoffmann. Thanks are due to Dr S. E. Read and Dr G. B. Whitfield, Jun., for gifts of lincomycin.

\section{REFERENCES}

ADAMS, M. H. (1959). Bacteriophages. New York: Interscience Publishers.

BANIC, S. (1959). Transduction to penicillin and chloramphenicol resistance in Salmonella typhimurium. Genetics 44, 449-457.

Barber, M. \& WATERWORTh, P. M. (1964). Antibacterial activity of lincomycin and pristinamycin: A comparison with erythromycin. British Medical Journal ii, 603-606.

BRYAN, B. E. (196I). Genetic modifiers of streptomycin resistance in pneumococcus. Journal of Bacteriology 82, 46I-470.

Demerec, M., Adelberg, E. A., Clark, A. J. \& Hartman, P. E. (I966). A proposal for a uniform nomenclature in bacterial genetics. Genetics 54, 6I-76.

Duncan, I. B. R. (1968). Development of lincomycin resistance by staphylococci. Antimicrobial Agents and Chemotherapy 1967, pp. 723-729.

GARROD, L. P. (1957). The erythromycin group of antibiotics. British Medical Journal ii, 57-63.

Griffith, R. S. \& BlaCK, H. R. (1970). Erythromycin. Medical Clinics of North America 54, I 199I2I5.

Gunsalus, I. C., Gunsalus, C. F., Chakrabarty, A. M., Sikes, S. \& Crawford, I. P. (1968). Fine structure mapping of the tryptophan genes in Pseudomonas putida. Genetics 60, 419-435.

Jones, W. F., Nichols, R. L. \& FinLAND, M. (1956). Development of resistance and cross-resistance in vitro to erythromycin, carbomycin, spiramycin, oleandomycin and streptogramin. Proceedings of the Society for Experimental Biology and Medicine 93, 388-393.

KJEMS, E. (1958). Studies on streptococcal bacteriophages. II. Adsorption, lysogenization, and onestep growth experiments. Acta pathologica et microbiologica scandinavica 42, 56-66.

Leonard, C. G., Colón, A. E. \& Cole, R. M. (1968). Transduction in group A streptococcus. Biochemical and Biophysical Research Communications 30, I30-I35. 
MALKE, H. (1967). Host-cell reactivation of ultraviolet-damaged phage in Streptococcus pyogenes. Biochemical and Biophysical Research Communications 29, 400-405.

MALKe, H. (1969). Transduction of Streptococcus pyogenes $\mathrm{k} 56$ by temperature-sensitive mutants of the transducing phage A25. Zeitschrift für Naturforschung 24b, 1556-1561.

MALKe, H. (1970). Characteristics of transducing group A streptococcal bacteriophages A5 and A25. Archiv für die gesamte Virusforschung 29, 44-49.

MCGEHEE, R. F., BARRETT, F. F. \& FINLAND, M. (1969). Resistance of Staphylococcus aureus to lincomycin, clinimycin, and erythromycin. Antimicrobial Agents and Chemotherapy 1968, pp. 392-397.

Paterson, P. Y. \& MAdDen, G. M. (1969). Occurrence and erythromycin susceptibility of penicillin resistant viridans streptococci in rheumatic fever patients on oral penicillin prophylaxis. Antimicrobial Agents and Chemotherapy 1968, pp. 323-325.

Pattee, P. A. \& Baldwin, J. N. (1962). Transduction of resistance to some macrolide antibiotics in Staphylococcus aureus. Journal of Bacteriology 84, $1049-1055$.

RAVIN, A. W. \& IYER, V. N. (196I). The genetic relationship and phenotypic expression of mutations endowing pneumoccocus with resistance to erythromycin. Journal of General Microbiology 26, 277-30I.

Rotheim, M. B. \& Ravin, A. W. (196I). The mapping of genetic loci affecting streptomycin resistance in pneumococcus. Genetics 46, 1619-1634.

SiRotNAK, F. M. \& HACHTEL, S. L. (1969). Increased dihydrofolate reductase synthesis in Diplococcus pneumoniae following translatable alteration of the structural gene. I. Genotype derivation and recombinational analyses. Genetics 6r, 293-3 2.

Weisblum, B. \& DemoHn, V. (1969). Erythromycin-inducible resistance in Staphylococcus aureus: Survey of antibiotic classes involved. Journal of Bacteriology $98,447-452$. 